\title{
Análisis metodológico de proyectos Fondecyt en antropología y sociología en 1992 y 1999
}

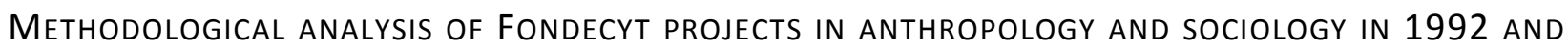
1999

Dra. Cecilia Millán (ceciliapazmlarivera@gmail.com) Programa APPeAL, Universidad Nacional Autónoma de México (Ciudad de México, México)

\begin{abstract}
The following paper is a descriptive and retrospective study that analyses the basic elements of a methodological design in projects approved by Fondecyt (one of the most important research funding agencies in Chile) in 1992 and 1999 considering the disciplines of anthropology and sociology. The analyses used four steps to create a methodological design. First, the projects selected were classified as qualitative or quantitative, according to the definition given by the own authors and looked for the arguments used by the authors for using the selected methodology. Then a detailed analysis is done on how these selected projects describe and elaborate a methodological strategy, reviling or not their stringency and precision of the methodological proposals.
\end{abstract}

Key word: fondecyt, methodological design, sociology, anthropology, coherence, stringency.

\section{Resumen}

El presente trabajo es un estudio descriptivo y retrospectivo que analiza los elementos básicos de un diseño metodológico en los proyectos aprobados por Fondecyt (una de las fuentes de financiamiento de investigación más importante en Chile) en los años 1992 y 1999 en dos disciplinas: antropología y sociología. Para ello se utilizó como criterio de análisis cuatro pasos para la elaboración de un diseño metodológico. Primero se definió a los proyectos como cualitativos o cuantitativos en el marco de la definición de los propios autores, buscándose también las argumentaciones dadas para el uso de una u otra metodología. Luego se realiza un análisis detallado acerca de cómo estos proyectos describen y elaboran una estrategia metodológica, develándose la rigurosidad y precisión de las propuestas metodológicas.

Palabras clave: fondecyt, diseño metodológico, sociología, antropología, coherencia, rigurosidad.

\section{Introducción}

Ruth Sautu en su libro Manual de Metodología, resalta la importancia del rol de la investigación en las ciencias sociales, sobre todo en sociedades complejas como las nuestras, donde diversos factores son los que influyen en la producción de la investigación en Latinoamérica. El texto es fruto del curso Teoría, objetivos y métodos de la investigación social que se dictó en el campo virtual de CLACSO a fines de 2004. 
El propósito del programa se desprendió de un diagnóstico que para la autora era casi innegable: "la preocupante debilidad teórica y metodológica cada vez más evidente en la producción de los científicos sociales, sobre todo, pero no de manera exclusiva, en las nuevas generaciones. Y que de cierta manera, se veían reflejados en proyectos de investigación incoherentes, en formulaciones imprecisas y en un irritante desorden argumentativo" (Sautu et. al. 2005:13).

Las reflexiones descritas han despertado la inquietud de retomar algunos de los puntos planteados y ver cómo se reflejan en la realidad chilena, realizando un diagnóstico de la metodología de estas investigaciones en una de las principales fuentes de financiamiento en Chile para realizar investigaciones (sobre todo en lo que respecta a las ciencias sociales): el programa Fondecyt.

Este trabajo evalúa las propuestas metodológicas presentadas en los proyectos de antropología y sociología que corresponden a los años 1992 y 1999. Primero, se describe qué metodología se utilizó, ya sea cuantitativa y/o cualitativa, y qué argumentaciones se dieron para el uso para el uso de una u otra metodología. Luego, se analiza el diseño metodológico utilizado por cada proyecto, desde la perspectiva y criterios planteados por Ruth Vieytes (2004). Finalmente, se hace un análisis comparativo entre los dos períodos y las dos disciplinas: antropología y sociología.

\section{Antecedentes institucionales de Fondecyt}

A partir de 1981 se crean en Chile fondos concursables a través de recursos públicos para las áreas de educación superior y desarrollo científico y tecnológico. Este fondo constituye hasta el día de hoy la principal fuente de recursos para el desarrollo científico y tecnológico del Chile, en cuanto a recursos asignados y el número de científicos que han participado.

Como se puede leer en su sitio web (www.conicyt.cl), el "Fondo Nacional de Desarrollo Científico y Tecnológico, Fondecyt, tiene por objetivo estimular y promover el desarrollo de investigación científica y tecnológica básica, y es el principal fondo de este tipo en el país. Creado en 1981, ha financiado más de 16 mil proyectos de investigación [hacia 2015] cuyos impactos han beneficiado tanto a la comunidad científica como a la sociedad en general". Estos fondos se dividen entre proyectos regulares, proyectos de iniciación en investigación y proyectos de postdoctorado.

Los proyectos regulares están orientados a investigadores con trayectoria. El investigador responsable puede participar junto a un grupo pequeño de co-investigadores y sus instituciones patrocinantes de pre y posgrado. El proyecto contempla financiamiento para personal (investigadores, personal técnico y tesistas), viajes de los investigadores, recursos para traer colaboradores extranjeros por un corto período, gastos de operación, equipamiento y apoyo a la infraestructura y gastos de administración para las instituciones patrocinantes. Los proyectos pueden tener una duración de dos a cuatro años y se financian en todas las áreas del conocimiento. La investigación se realiza en el país.

Los proyectos de iniciación en investigación están orientados a investigadores jóvenes que han obtenido el grado de doctor en los últimos cinco años. El investigador responsable, junto a su institución patrocinante, obtiene financiamiento para personal técnico y tesistas. El proyecto contempla los mismos ítems financiables que en el concurso regular. Pueden tener una duración de dos a tres años y se financian en todas las áreas del conocimiento. Se entrega por única vez al investigador y la investigación se realiza en el país. 
Los proyectos de postdoctorado están orientados a investigadores jóvenes que han obtenido el grado de doctor en los últimos tres años. El investigador responsable debe contar con un investigador patrocinante y una institución patrocinante. El proyecto contempla honorarios para el postdoctorante, apoyo para la investigación, beneficio de salud y gastos de instalación en caso que sean necesarios. Los proyectos pueden tener una duración de dos a tres años y se financian en todas las áreas del conocimiento. Este beneficio se entrega por única vez al investigador. La investigación se realiza en el país.

Como antecedente general, con respecto a los concursos Fondecyt regular según tipos de ciencias, en el año 1992 fueron aprobados en el área de ciencias sociales 42 proyectos y en el área de ciencias exactas y naturales 133 proyectos. En cambio en 1999 para las ciencias sociales fueron aprobados 40 proyectos y en las ciencias exactas y naturales 146 proyectos. Lo cual refleja que los proyectos del área de las ciencias exactas y naturales en estos períodos fueron en aumento, no así en las ciencias sociales, existiendo aún diferencias importantes entre el financiamiento de un área y otra.

\section{Paradigma y metodología: dicotomía cualitativo - cuantitativo}

Hay posturas que sostienen que para abordar de una manera científica un problema metodológico se debe analizar qué modelo o enfoque conceptual es el más adecuado para enfrentar con éxito una investigación. Por lo tanto, se sugiere analizar los distintos modelos conceptuales con la finalidad de tener un criterio fundado para optar por uno u otro paradigma y diseñar la estrategia que mejor convenga para los objetivos de la investigación. Se puede sostener que el paradigma que se adopte va a condicionar los procedimientos de estudio que se sigan en la investigación. De alguna manera, cada paradigma ya sea cualitativo o cuantitativo mantiene una concepción diferente de lo que es la investigación, cómo investigar, qué investigar y para qué sirve la investigación.

Aunque no existe una única definición de paradigma, existe cierto grado de consenso al respecto, entendiéndose, entonces "como un conjunto de creencias y actitudes, una visión del mundo compartida por un grupo de científicos que implica metodologías determinadas" (Pérez 2001:17).

Sin embargo, desde la mirada epistemológica de Gloria Pérez podemos señalar que la filosofía de la ciencia, en la actualidad, no admite la existencia de criterios absolutos de demarcación del criterio de ciencia. Por el contrario, se acepta que son las comunidades científicas las que justifican y validan la ciencia según las relaciones que se establecen entre el método empleado, el conocimiento obtenido y el contexto en el que uno u otro se dé. Por lo tanto, según el concepto de paradigma que tenga una determinada comunidad científica, la investigación que se realice tendrá características particulares.

Desde esta perspectiva, según la misma autora, hay que hacer notar que carece de base suficiente el debate a nivel epistemológico entre los paradigmas que hasta ahora se han utilizado en apoyo a la investigación, el racionalista y el empirista, pues hay que aceptar que cada paradigma descansa sobre ciertos supuestos que se deben comprobar en el contexto de su aplicación. De esta manera, es adecuado seleccionar aquel cuyos supuestos se acomoden mejor al fenómeno que se está investigando, lo que no significa que los métodos particulares estén necesariamente vinculados a un paradigma.

Dado lo anterior, resulta pertinente preguntarse una serie de cuestiones metodológicas, una de ellas es si los paradigmas exigen por su "naturaleza" el uso de ciertos métodos y no otros de investigación. En otras palabras, si existe inconsistencia entre la filosofía de un paradigma y el uso de los métodos que tradicionalmente se han asociado a otro paradigma. 
Autores como Cook y Reichardt (2005) sostienen que existe una falacia entre el debate y el conflicto entre paradigmas metodológicos, que se sostiene en la idea de que un tipo de método se asocia inexorablemente a un paradigma. Esta visión es rígida y crea una exclusión de las metodologías según el paradigma, siendo imposible el uso de ambos.

Frente a esta posición de incompatibilidad, ambos autores se hacen una serie de preguntas para demostrar lo erróneo de la postura tajante entre los paradigmas metodológicos. Se preguntan, por ejemplo, si quien utiliza métodos cuantitativos es necesariamente positivista y si quien utiliza métodos cualitativos es fenomenólogo. A través, de diversos ejemplos de investigaciones los autores demuestran que esta exclusión es errónea y limitada, ya que la investigación puede nutrirse de un planteamiento fenomenológico con un enfoque cualitativo y utilizar además métodos cuantitativos o a la inversa. Asimismo, frente a la afirmación de que un procedimiento cualitativo es per se subjetivo y uno cuantitativo es objetivo, argumentan que si lo subjetivo se entiende como las influencias que tiene el ser humano sobre los hechos, ambas técnicas son subjetivas. Si éste se entiende como los sentimientos y creencias que median en la investigación, en ambas técnicas existen grados de subjetividad, no siendo monopolio de las técnicas cualitativas. Del mismo modo, sobre la diferencia en cuanto a ser lo cualitativo exploratorio e inductivo versus lo cuantitativo confirmatorio y deductivo, argumentan que ambos caminos permiten generar teoría así como comprobar datos. Son atribuciones que se extienden más allá de los métodos (Cook y Reichardt 2005).

A partir de lo señalado, si bien hay ciertos métodos que se articulan de mejor modo con un paradigma que otro, no es correcto asociarlo con un solo tipo de método ya que no determina una única elección de métodos. Lo importante es el objeto de estudio, que exigirá el privilegio de ciertos métodos por sobre otro o su combinación.

Al respecto Ruth Vieytes (2004) señala que además de la diversidad de posturas, existe una denuncia mutua. En cuanto a ambos extremos de la postura, quienes defienden los diseños cuantitativos consideran lo cualitativo una mera información previa de la investigación, que es útil para identificar variables y definir problemas, a través de entrevistas o grupos focales, todo bajo el rotulo de investigación exploratoria, pero cuando la investigación es concluyente se utilizan solo las herramientas cuantitativas. Para este tipo de investigador, la perspectiva cualitativa no existe y se expresa en su silencio. En el otro polo, consideran lo cuantitativo inútil y carente de sentido, considerando que son incompatibles la combinación de metodologías. Frente a esta polémica, la autora señala que lo importante es la naturaleza del problema de investigación y las condiciones del contexto en el que va a desarrollarse.

Frente a la supuesta incompatibilidad, hay autores que reconocen las ventajas que tiene el uso del conjunto de métodos. Por ejemplo, es pertinente en el caso de tener objetivos múltiples, ya que el uso de ambos métodos enriquece el proceso de investigación, además su combinación permite que se complementen los aportes particulares de cada método y contribuye a corregir los sesgos inevitables de cada uno, pudiendo ser la triangulación una fortaleza.

Salvador Perelló (2009), quien considera estéril la polémica entre cuantitivistas y cualitivistas, señala que no se trata de subordinar una estrategia metodológica a otra, sino más bien, articularlas en un mismo proyecto de investigación. Esta perspectiva metodológica a partir de los 80 's se ha denominado triangulación y consiste en la utilización de distintos métodos en una misma investigación. La triangulación, en general, puede efectuarse en distintos niveles: datos, investigadores, teorías o los métodos que se empleen para recoger datos. 
La triangulación metodológica, que consiste en la combinación de métodos de investigación, tiene la ventaja de potenciar la validez de los hallazgos ya que se evita que lo "descubierto" sea creación del método que se está utilizando y aporta en potenciar la investigación debido que ningún método por sí mismo es un medio universal para comprender los fenómenos de la realidad social (Perelló 2009).

Sobre la dicotomía cuantitativa y cualitativa, Gloria Pérez señala que "al distinguir entre paradigma/método como niveles diferentes de decisión, cabe destacar las siguientes consideraciones:

1. La opción por un paradigma determinado no es exclusiva del método elegido. No se contradicen, por el contrario pueden complementarse.

2. Si algún investigador lleva a cabo una investigación cualitativa, no tiene por qué asumir todos los atributos del paradigma en cuestión.

3. La mayoría de los investigadores optan por un paradigma de tipo fenomenológico, con independencia de que orienten su investigación hacia un proceso o resultado.

4. Los métodos cualitativo/cuantitativo pueden aplicarse conjuntamente según las exigencias de la situación investigada.

5. La utilización conjunta no está exenta de dificultades. De ahí que nos sirva de estímulo saber que existe una actitud integradora de ambas posturas" (Pérez 2001:31).

Los argumentos dados por los autores reflejan una polémica que carece de sentido sobre los métodos y técnicas de investigación y que sería importante superar en post del desarrollo científico. De acuerdo a lo planteado, la polémica de método cualitativo / cuantitativo es falaz ya que identifica "cada método con un paradigma distinto y opuesto, se afirma la radical oposición entre ambos paradigmas a la vista de unos presupuestos meta teóricos y se oculta la posibilidad de utilización conjunta de los métodos cualitativos y cuantitativos" (Pérez 2001:32). Por otra parte, "no existe nada, excepto quizás la tradición, que impida al investigador mezclar y acomodar los atributos de los dos paradigmas para lograr la combinación que resulte más adecuada al problema de investigación y al medio con que se cuenta" (Cook y Reichardt 2005:40).

De la discusión y argumentos se concluye que hay un error en asociar dos niveles que deberían mantenerse separados: el nivel paradigmático y el nivel metodológico/técnico. Lo que se propone es que no exista una oposición entre los dos métodos y, en cambio, exista una complementariedad si el problema de investigación lo amerita y exige, es decir, se puede elegir el método sin considerar el paradigma como determinante de su elección.

\section{Los tipos de investigación social: diseño metodológico}

El proceso de investigación conlleva tomar diversas decisiones que abarcan aspectos epistemológicos, teóricos, metodológicos y técnicos.

El momento epistémico es la etapa en que el investigador construye su objeto de investigación, lo que hace a partir de un cuestionamiento a lo que aparece como dado. Es la etapa en que se construye el objeto de estudio y que involucra seleccionar tema de investigación, plantear el problema, elaborar el marco teórico y formular las hipótesis y objetivos (Vieytes 2004).

Otro momento es el técnico metodológico que implica decidir el cómo investigar, es decir, qué estrategias y técnicas se utilizarán: "es el proceso en que se deciden y desarrollan las estrategias del encuentro con la 
realidad" (Vieytes 2004:19). Se selecciona el diseño, se define el universo, muestra y unidad de análisis, se preparan los instrumentos, se recolectan y analizan los datos. Este proceso no se reduce solo a las metodologías y técnicas ya que éstas se articulan tanto con la teoría como con las decisiones epistemológicas. Es decir, cada técnica o metodología responde al objeto de estudio y a una mirada de la realidad.

En el último momento, que es el teórico, se interpretan los resultados, se sintetizan las conclusiones y se elabora el reporte de investigación (Vieytes 2004). Cabe mencionar que las etapas descritas no son lineales, sino que se articulan e intercalan según avance el proceso de investigación.

Si observamos el proceso de investigación, éste al igual que cualquier tipo de objeto o conjunto de objetos puede ordenarse según alguna regularidad que presente, y si ocurre que las regularidades son de distinta naturaleza, darán lugar a distintas clasificaciones. Estas distinciones nos permiten definir los tipos de investigación. Es así que si se utiliza el criterio de finalidad de la investigación, se puede clasificar entre: investigación básica, aplicada e investigación tecnológica; según el diseño investigación: primaria o secundaria; según la estrategia teórico-metodológica: cuantitativa o cualitativa; de acuerdo a los objetivos: exploratoria, descriptiva, correlacional o explicativa; por el grado de control de las variables: experimental o no experimental y por la temporalidad: transversal (sincrónica) o longitudinal (diacrónica) (Vieytes 2004).

Respecto al análisis presente en este artículo, el trabajo se centra en el criterio "estrategia" o "diseño metodológico" planteado por Ruth Vieytes, que responde a los objetivos de este trabajo que es analizar el diseño metodológico de las propuestas de investigación y la opción estrategia teórico-metodológica: cualitativa o cuantitativa.

Para Hernández, Fernández y Baptista (2004) el diseño metodológico es una manera práctica de responder a las preguntas de investigación. De alguna manera implica desarrollar un diseño de investigación y aplicarlo al contexto particular del estudio. Este plan incluye actividades que apunten a encontrar las respuestas a las preguntas de la investigación. Sin embargo, las alternativas a crear una u otra estrategia depende de lo que el investigador determine como óptimo para responder de mejor forma a la pregunta de investigación.

Para Guillermo Briones (1989), el diseño de investigación consiste en formular un plan para llevar a cabo lo que se haya planteado a partir del problema de investigación y de sus correspondientes objetivos. Un diseño de investigación, es la estrategia para cumplir con la investigación que se ha propuesto el investigador; estrategia que a nivel concreto resulta de una serie de decisiones tomadas frente a diversas alternativas en una cadena de medios o posibilidades para alcanzar los objetivos. Tales decisiones finalmente se transforman en una serie de actividades y tareas de muy diverso tipo.

Según Briones, los pasos que forman parte de los diseños en las investigaciones tradicionales son: 1) Plan para el análisis de contexto: toda investigación tiene dos orígenes principales, por un lado la zona temática, ya estudiada por otros autores y sobre la cual puede existir una o varias teorías que surgen del resultado de las investigaciones y, por otro lado, una situación social que requiere algún tipo de solución o de modificación. Ambos orígenes pueden relacionarse de diferentes maneras y a diversos niveles. 2) Definición del universo de estudio: es necesario delimitar o definir con claridad el grupo o conjunto de personas o la categoría social al cual se refiere dicho estudio. De igual manera el contexto geográfico y el ambiente en el cual se encuentra. 3) Selección de las unidades de análisis: las unidades de análisis son las personas u objetos de los cuales se tomará la información y a las cuales se referirá el informe de 
investigación. 4) Recolección de información: debe indicarse cómo se recogerá la información, lo cual está estrechamente relacionado con el problema de investigación y de los objetivos de ésta. 5) Pruebas de procedimientos para la recolección de información: antes de explicar los procedimientos o técnicas elegidas es necesario probar los mismos con un grupo de personas de la población a estudiar o en personas con características similares. 6) Recolección de la información en terreno (trabajo de campo): el diseño de la investigación deberá pensar cómo debe procederse en el terreno para recoger la información con los procedimientos ya probados. 7) Procesamiento y análisis de la información recogida: la última decisión sustantiva que debe tomarse al diseñar una investigación se refiere a los procedimientos que se utilizarán en el procesamiento y análisis de los datos.

Para Ruth Vieytes el diseño "hace referencia a una porción francamente reducida del proceso de investigación, aunque decisiva puesto que se ubica en el núcleo de las operaciones que transforman el mero consumo de conocimientos previo o la especulación filosófica, en un dispositivo de conexión de ese conocimiento previo con la información existente fuera de él: en la realidad misma" (2004:16).

Para esta autora, seleccionar el diseño de una investigación corresponde a un momento técnicometodológico. Si el objeto de la investigación ya es producto o resultado de una construcción, ese proceso exige pensar en algún tipo de plan para llegar a él. Ahora, lo particular del diseño de la investigación es que se trata de un plan que debe permitir al investigador salir del ámbito especulativo y le debe permitir que funcione el modelo en la realidad social.

Hay varios diseños o estrategias a utilizar, algunos recurrentes en las ciencias sociales o humanas, siendo una condición estudiarlos todos, pues los criterios para optar por uno o por otro diseño pueden ser erróneos si no hay fuertes fundamentos epistemológicos y metodológicos que permitan un vínculo coherente entre lo que el investigador pretende averiguar y el cómo lograrlo.

Ahora bien, la interrogante es saber cuál es el mejor diseño para una investigación y la respuesta no es fácil ni precisa. Para Vieytes "un buen diseño es aquel que combina buenos criterios de recolección de datos y de análisis de los mismos, siempre desde la perspectiva de los objetivos y las hipótesis" (2004:27), haciéndose cargo de éstos. Por lo tanto, la validez de una investigación estará determinada por el grado de congruencia del diseño elegido con el problema y con las conjeturas que se ponen a prueba. Es importante señalar que un diseño u otro no tienen en sí mismo valor científico, sino que se concede el estatus científico a la compleja adecuación entre los procedimientos a seguir con los enunciados a verificar.

El diseño o estrategia de investigación, según Ruth Vieytes, debe contemplar los siguientes cuatro pasos:

(1) Definir el universo, las muestras y las unidades: en tanto no podemos estudiar a todas las personas, situaciones o fenómenos, debemos hacer accesible el universo determinando las muestras. De los objetivos y del diseño que utilice el investigador, deberá derivarse la elección de los sujetos a estudiar (los elementos, personas, fenómenos que constituyen la muestra de la investigación). Estos elementos forman parte de un grupo de conceptos, que se definen como el universo, "que es una serie real o hipotética de elementos que comparten características definidas, relacionadas con el problema de la investigación; una población que es un conjunto definido, limitado y accesible del universo, que forma el referente para la elección de la muestra, es el grupo al cual se intenta generalizar los resultados del estudio y la muestra que es el conjunto de individuos extraídos de la población a partir de algún procedimiento científico" (2004:27). 
(2) La preparación de los instrumentos, que son las diferentes tecnologías de "recolección de datos". Esta etapa incluye tanto la operacionalización de los conceptos como la elaboración del instrumento. Inicialmente la forma en que utilizamos el modelo teórico es dando a sus términos un carácter netamente conceptual, pero de alguna manera hay que hacerlos operativos y convertirlos en variables observables. "Al operacionalizar estos términos, especificamos cuáles serán las manifestaciones concretas y observables de esos conceptos que nos permitan valorarlos. Se denomina a esas expresiones empíricas indicadores y constituyen un elemento imprescindible para poder elaborar el instrumento" (2004:28). Luego, la elaboración del instrumento, que es un dispositivo que se utiliza para medir, debe permitir nombrar, comparar, clasificar o cuantificar y debe ser pensado como un procedimiento de medición ya sea en estudios teóricos o empíricos.

(3) La recolección de los datos se realiza una vez establecida una estrategia global de contrastación empírica, donde ya se ha operacionalizado el universo, las hipótesis y se han elegido o construido los instrumentos con los que se van a recolectar los datos, por lo tanto se da comienzo a la tarea de recolección y registro de los datos.

(4) Luego se analizan los datos, que deben ser codificados. "Codificar significa asociar un número a cada categoría de respuestas a los efectos del conteo o del cómputo... los datos se seleccionan, se ordenan, se jerarquizan según algún principio lógico que permita hacerlos operativos" (2004:29). En el análisis de datos con técnicas cuantitativas se debe explicitar que técnica se utilizará y por qué, además de qué resultados se espera obtener. En el caso del análisis cualitativo el proceso de análisis comienza mucho antes.

Se continúa con la etapa que Ruth Vieytes denomina momento teórico, que incluye interpretación, conclusiones y comunicación. En esta etapa se interpretan los resultados, proceso en el cual el investigador evalúa la propuesta teórica a la luz de los resultados y vincula sus conclusiones con el conocimiento disponible hasta el momento sobre el problema. Una vez hecho lo anterior, hay que tener presente que siempre queda una oportunidad para inferir consecuencias o causas no manifiestas acerca de lo no constatado pero, sí sugerido por el análisis. Luego se sintetizan las conclusiones, que es la expresión de toda la labor realizada que concluye en ciertas y específicas conclusiones que deben escribirse de "una manera sintética y concentrada en torno a lo que se demostró en el análisis. Esta actividad [...] es indispensable, pues no todos los potenciales destinatarios pueden estar interesados en conocer el proceso completo que se ha realizado y por ello el investigador debe ofrecer sus conclusiones de manera escueta y simple" (2004:30). Finalmente, se elabora el informe o reporte de investigación, que es el producto final de proceso de investigación y que debe resumir en forma clara los resultados logrados. Cuando los fines de la investigación son aplicados, puede agregarse un apartado denominado recomendaciones, las cuales se refieren a lo que en la etapa de planteo del problema se ha denominado "problema práctico".

De acuerdo a lo anterior, la propuesta hecha por Ruth Vieytes es bastante completa y flexible, ya que propone una estrategia metodológica sin distinciones de paradigmas, enfoques, ni técnicas. Además, da cuenta del proceso complejo de la investigación y de la necesaria articulación de los diversos momentos, de la necesaria rigurosidad y transparencia de cada etapa. Asimismo, en su texto incluye y desarrolla dimensiones de la investigación que suelen no ser consideradas y profundizadas en los diversos manuales y libros de metodología, como por ejemplo, la distinción e importancia entre datos primarios y datos secundarios para la investigación. 
Como última consideración es importante señalar, que tal como lo indica el siguiente cuadro, aunque exista alguna especialización, las técnicas pueden ser utilizadas en relación al conjunto de métodos cualitativos y cuantitativos.

Cuadro 1

Métodos y técnicas de producción de datos según diferentes estrategias metodológicas

\begin{tabular}{|c|c|c|}
\hline Metodología & Métodos & Técnicas de producción \\
\hline Cuantitativa & $\begin{array}{l}\text { Experimental } \\
\text { Encuesta } \\
\text { Análisis cuantitativo de datos } \\
\text { segundarios (estadística) }\end{array}$ & $\begin{array}{l}\text { Cuestionarios } \\
\text { Recopilación de datos existentes } \\
\text { (censos, encuestas, estadísticas } \\
\text { continuas) } \\
\text { Análisis de contenido de documento, } \\
\text { textos, films, etc. }\end{array}$ \\
\hline Cualitativa & $\begin{array}{l}\text { Etnográfico } \\
\text { Análisis cultural } \\
\text { Estudio de caso/s } \\
\text { Biográfico } \\
\text { Análisis de conversaciones } \\
\text { Grupos Focales }\end{array}$ & $\begin{array}{l}\text { Entrevistas interpretativas } \\
\text { Entrevistas etnográficas } \\
\text { Observación no participante } \\
\text { Observación participante } \\
\text { Análisis de documentos } \\
\text { Análisis de material visual/auditivo }\end{array}$ \\
\hline
\end{tabular}

Nota: las técnicas son aplicables a varios métodos. Fuente: Sautu, Boniolo, Dalle y Elbert (2005:47).

\section{Diseño metodológico}

El interés inicial fue analizar dos disciplinas de las ciencias sociales: antropología y sociología, en tres periodos históricos. El criterio utilizado sería el primer año de aprobación de proyectos Fondecyt en 1982, luego un año intermedio (1994) y un año reciente de proyectos aprobados (2007). Dicha idea no se concretó debido a que en el primer año de aprobación, se adjudicó un proyecto antropología y ninguno para sociología, lo cual obligó a modificar los criterios iniciales. Se optó por revisar los proyectos del año 1992 y 1999 de las respectivas disciplinas que se encontraban en el Archivo Nacional de Chile. Los proyectos adjudicados llegan solo al Archivo Nacional después de terminado su proceso de ejecución, única institución donde se puede tener acceso a las propuestas que hacen los distintos investigadores a los proyectos Fondecyt en Chile.

El presente trabajo es un estudio descriptivo, realizado de forma retrospectiva abocándose a los proyectos aprobados por Fondecyt en 1992 y 1999. Primero, se describe qué metodología se utilizó en las disciplinas de sociología y antropología y las argumentaciones que se dieron para el uso de una u otra metodología. Luego se realiza un análisis comparativo entre los dos años y entre ambas disciplinas. Finalmente, se analiza el diseño metodológico utilizado por cada proyecto desde la perspectiva y criterios planteados por Ruth Vieytes. En este apartado se describen y analizan los pasos que han sido considerados dentro de los diseños de investigación de los proyectos Fondecyt, es decir, se busca revisar qué aspectos son considerados y cuáles no, en el proceso metodológico de cada investigación, según los criterios establecidos por Vieytes.

Cabe destacar que Fondecyt en el ítem metodología exige los siguientes términos: "Metodología: Describa los métodos que planea utilizar para abordar cada uno de los objetivos del proyecto (por ejemplo describa las técnicas experimentales, justifique los tamaños muéstrales, precise los análisis estadísticos, etc.). La 
extensión mínima de esta sección es de 3 páginas". Este ítem es lo que se exigía el año 2007 (que es cuando se realiza este estudio). Para los proyectos del año 2015 se exigió un máximo de 12 páginas para la formulación del proyecto, que incluye la metodología, además de la formulación de la propuesta, hipótesis, objetivos generales y específicos, plan de trabajo, trabajo adelantado y recursos disponibles. Solo la sección de bibliografía tiene un máximo de 3 páginas (un archivo independiente a la formulación del proyecto). Resulta destacable que cada vez se ha ido disminuyendo el espacio para el desarrollo de la metodología de la investigación, que hoy se reduce a dos páginas, en el caso de que se distribuyan equitativamente los aspectos solicitados en la formulación del proyecto.

Dos son los proyectos aprobados en antropología el año 1992: 1) Bienestar psicosocial y relaciones interpersonales de los adultos mayores. Siendo la investigadora responsable Carmen Barros Lezaeta $\left(\mathrm{N}^{\circ}\right.$ 1990843) y 2) Investigación antropológica cultural relativa a colección etnográfica institucional de la etnia mapuche. El investigador responsable es Héctor Zumaeta Zuñiga ( $\left.N^{\circ} 1990004\right)$.

En la misma disciplina, el año 1999 se aprobaron dos proyectos. 1) Simbolizaciones de la diferencia sexual en la religiosidad de los Aymaras del norte chileno. La investigadora responsable es Vivian Gavilán Vega ( $\left.N^{\circ} 1990200\right)$ y 2) Etnias, identidades colectivas y estados nacionales en el norte de Chile (S.XIX-XX). El investigador responsable es Hans Gunderman Kroll ( $\left.N^{\circ} 1990503\right)$.

En sociología el año 1992 se aprobaron cuatro proyectos. 1) Estudio de factibilidad de un programa concertacional de salud mental a nivel de la comuna. Investigador responsable Rafael Eduardo Sepúlveda Jara ( $\left.N^{\circ} 1990431\right)$. 2) Transformaciones agrarias, familia y mujer campesina. La investigadora responsable es Jimena Valdés Subercaseaux ( $\mathrm{N}^{\circ}$ 1990415). 3) Problemas ambientales a nivel comunal en Chile: alternativas para acciones de la comunidad y de las municipalidades. El investigador responsable es Ernst Hajek Girardi ( $N^{\circ} 1990639$ ) y 4) Jóvenes pobladores y organizaciones comunitarias: estudio de caso en la Florida. El investigador principal es Francisco Sabatini Downey ( $N^{\circ} 1990613$ ).

En la misma disciplina el año 1999 se aprobaron siete proyectos. 1) El peso del capital social en los procesos de movilidad social en la década del noventa. Análisis comparativo de los casos de Chile, Argentina y Uruguay. El investigador responsable es Vicente Espinoza Espinoza ( $\left.\mathrm{N}^{\circ} 1990818\right)$. 2) La familia del adulto mayor: composición e interrelaciones. La investigadora responsable es Carmen Barros Lezaeta $\left(\mathrm{N}^{\circ}\right.$ 1990562). 3) El impacto de la violencia de género en una comunidad urbana. La Serena 1900-1925. Investigador responsable María Celina Tuozzo Bourrier (N 1990163). 4) Trabajo temporero, flexibilidad laboral y productividad en la empresa subcontratista de la VIII región. Un estudio sobre los rezagos en la modernización socioeconómica de la actividad forestal. Investigador responsable José Aquevedo Soto ( $\mathrm{N}^{\circ}$ 1990383). 5) Macro y micro efectos sobre las decisiones reproductivas: una modelización multi-niveles de los conductos anticonceptivos de mujeres fértiles de la octava región de Chile. Investigador responsable es José Manuel Merino Escobar ( $\left.N^{\circ} 1990392\right)$. 6) Pobreza y medio ambiente: estudios de casos en la zona central de Chile. El investigador responsable es Ernst Hayek Girardi ( $\left.N^{\circ} 1990162\right)$ y 7 ) La influencia de las mujeres en las trayectorias laborales e identidad masculina. La investigadora responsable es Virginia Guzmán Barcos ( $\left.N^{\circ} 1990140\right)$. 


\section{Resultados}

\section{Tipo de investigación según diseño metodológico por disciplina y por año}

El criterio utilizado para definir el tipo de investigación, cuantitativo o cualitativo, fue en primera instancia el de los propios investigadores. En caso de no ser explicitado, se definió bajo un análisis de la coherencia de cada investigación en términos de enfoque, variable a medir, su operacionalización, las técnicas utilizadas y los tipos de análisis que se proponen. Al analizar la totalidad de los proyectos se observa lo siguiente.

De los catorce proyectos analizados sólo nueve explicitan el tipo de diseño que están utilizando, ya sea cuantitativo y/o cualitativo. De estos nueve proyectos, hay seis que explicitan el uso de las dos metodologías, es decir, un diseño metodológico cuantitativo y cualitativo. Los otros tres describen su estrategia metodológica como cualitativa.

Los restantes cinco proyectos no explicitan el tipo de diseño que utilizan, por lo cual fue definido según el criterio de quien escribe este trabajo, tres proyectos tienen un enfoque cualitativo y dos cuantitativo.

De lo anterior, se puede inferir que la mayoría de las investigaciones fueron explícitas en señalar el tipo de diseño metodológico que estaban utilizando, lo que es relevante debido a la importancia de la transparencia en todo tipo de investigación, que permite saber con detenimiento desde qué perspectiva y enfoque están trabajando los autores. En términos comparativos los proyectos sociológicos en su mayoría explicitan el tipo de diseño (siete de diez), en cambio los antropológicos solo lo hacen la mitad (dos de cuatro).

Por otra parte, los resultados muestran, sin hacer distinción entre años y disciplina, que lo que más se utiliza de manera similar es la combinación de diseño (seis proyectos utilizaron diseño cuantitativo y cualitativo) y el diseño cualitativo (seis proyectos). En cambio el diseño cuantitativo por sí solo es menos utilizado (dos proyectos). De lo anterior, se puede inferir que las opciones metodológicas obedecen a las características del objeto de estudio.

Si analizamos por disciplina, encontramos que los cuatro proyectos aprobados para antropología optaron por un diseño cualitativo. En cambio en sociología, de los diez, uno es cuantitativo, dos son cualitativos y seis combinan ambas metodologías, lo que refleja una mayor apertura al uso de ambas metodologías. Suponemos que esto no sucede en antropología debido a que esta ciencia tradicionalmente se ha vinculado a técnicas que se clasifican dentro del diseño cualitativo, como son las entrevistas, observaciones y técnicas etnográficas. Desde esa perspectiva, la sociología ha estado más vinculada a técnicas como encuestas y observación estructurada, lo que podría explicar la elección. En esta misma disciplina el uso de lo cualitativo no queda excluido, ya que desde las ciencias sociales en general este tipo de diseño ha sido más valorado, en términos de entregar mayor profundidad y comprensión de la realidad social.

Razones que dan los investigadores para justificar el tipo de investigación cuantitativa y/o cualitativa según diseño metodológico

Con respecto a los motivos que llevan a los investigadores a utilizar un diseño u otro, solo se explicita en uno de los cuatro proyectos de la disciplina antropológica y cuatro de los diez en sociología. Lo descrito 
muestra lo poco relevante que resulta explicitar la posición epistemológica y metodológica del investigador, lo cual es fundamental para transparentar el proceso de investigación.

En el proyecto de antropología de 1999, solo se señala a favor del uso del diseño cualitativo "que se debe principalmente al objeto de estudio, el cual tiene características que hace que sea mejor analizado y abordado desde una mirada cualitativa y no así cuantitativa". Esta frase no da argumentos de por qué sería mejor un diseño cualitativo por sobre uno cuantitativo.

En el caso de sociología, en 1992 tenemos que la opción metodológica es mixta, es decir, utiliza tanto lo cuantitativo como lo cualitativo, sin embargo, más que dar razones por las cuales utiliza ambos diseños, hace hincapié en que su objeto de estudio son las mujeres y familias y que es un tema que ha sido más tratado en fuentes de carácter cualitativo que cuantitativo, por lo cual se privilegió lo cualitativo.

Finalmente, en el año 1999 en sociología la opción metodológica cualitativa se debe a la importancia de los significados que dan los sujetos a la realidad social, lo cual desde los autores es más acorde a ese tipo de diseño. Los otros dos proyectos que explicitan las razones optaron por un diseño mixto. Al respecto, uno de ellos divide la investigación en dos niveles. Uno cuantitativo, señalando que les permite analizar las estructuras y que se utilizará lo cualitativo cuando se aboquen a la percepción de los sujetos de estudio. Ambas opciones dan cuenta de los énfasis de cada diseño, dependiendo la opción de uno u otro del objeto de estudio. El segundo proyecto argumenta algo similar al anterior, dividiendo en dos fases la metodología, una cuantitativa que tiene que ver con datos macroestructurales y una cualitativa que busca comprender. En este sentido ambas argumentaciones apuntan a las concepciones tradicionales de lo cuantitativo y cualitativo, relacionando lo cualitativo a la comprensión versus cuantitativo a la explicación, como también lo cualitativo a lo micro versus lo macro que sería cuantitativo.

\section{Revisión del diseño metodológico}

Antropología 1992. Con respecto al diseño metodológico por disciplina y año, se constata que en los dos proyectos aprobados en 1992 en antropología, en relación al universo, muestra y unidades, solo un proyecto, 1990843, explica cuál es el universo y la muestra, siendo justificado el universo y explicándose en detalle la muestra, sus criterios y las razones de su elección. El otro proyecto describe la muestra en términos generales, sin mencionar cómo se define y bajo qué criterios. Con respecto a la preparación de instrumentos, en ambos casos no se da cuenta de la operacionalización de conceptos, solo se explicitan las técnicas a utilizar mencionando de manera breve sus ventajas. Luego, en la recolección de datos, un proyecto, 1990843, precisa la forma en que se recolectarán los datos, no así en el otro, que no precisa cómo se recolectará la información, pero sí a través de qué medios se registrarán los datos (diario de campo, cintas magnetofónicas, fotografías y diapositivas). En cuanto al análisis de datos, no están contendidos en el apartado metodológico de ambos proyectos.

Antropología 1999. En relación a los dos proyectos de antropología aprobados en 1999, al analizar el universo, muestra y unidades, en el proyecto 1990503 no se menciona el universo, la muestra, ni las unidades. La muestra sólo se infiere a partir de la descripción de las técnicas que se utilizarán. Por lo tanto, no queda clara la muestra, ni el criterio de selección mencionado. En el segundo proyecto, 1990200, se menciona que el estudio de caso se abocará a "la población aymara de comunidades altiplánicas del sector norte", lo cual lleva a pensar que se refiere a la muestra. Luego, en el apartado de técnicas y procedimientos, se explicita a los sujetos de estudio para las entrevistas e historias de vida. No se explicita claramente el criterio de selección mencionado, sólo en el segundo caso se señala que se considerará la 
variable "generacional y adscripción religiosa". Con respecto a la preparación del instrumento, en ambos proyectos no se da cuenta de la operacionalización de conceptos. Se explicita en ambos casos la técnica que se utilizará, argumentando sus ventajas y no especificando la elaboración del instrumento. Luego, en la recolección de datos, no se da cuenta en ninguno de los dos proyectos cómo se recolectarán los datos, ni cómo se registran. En cuanto al análisis de datos, solo un proyecto, 1990503, señala que realizará una "triangulación" para así aumentar las condiciones de validez de los resultados.

Sociología 1992. Con respecto al diseño metodológico por disciplina y año, se constata que en uno, 1990639, de los cuatro proyectos aprobados en 1992 no se logró obtener el diseño metodológico correspondiente a esta investigación. La causa se desconoce, es posible suponer que se haya extraviado, pues sería bastante poco rigurosa la aprobación de un proyecto Fondecyt sin su apartado metodológico. El documento obtenido describe un taller-seminario, pero no un diseño metodológico. En relación a los otros tres proyectos aprobados, con respecto al universo, muestra y unidades que el proyecto 1990431 explicita su muestra, pero no señala los criterios y las razones de dicha muestra. Respecto al segundo proyecto 1990415 se explicita el universo, sin embargo, la muestra no queda claramente definida, pues indica que son familias de estas localidades, pero no indica número, ni criterios para escogerlas, sí explicitándose las unidades de análisis. En el tercer proyecto 1990613 se da cuenta de modo muy genérico del grupo que se quiere estudiar y que se deduce corresponde a la muestra. En relación a la preparación de instrumentos, el proyecto 1990431 no menciona la operacionalización de conceptos, pero sí el tipo de técnicas que se utilizarán, especificando para ambas técnicas lo que se pretende medir. El proyecto 1990415 no indica claramente cuál es el procedimiento, pero indica de qué tipo es y conceptualiza un par de distinciones que parecen relevantes a la hora de recoger la información. Por último, el proyecto 1990613 señala solo la técnica que se utilizará, que es la entrevista en profundidad y no señala la operacionalización de los conceptos, ni tampoco cómo se elaboró la técnica. Con respecto a la recolección de datos, el proyecto 1990431 no hace ninguna mención. El proyecto 1990613 menciona de forma general que se realizará a través de bases estadísticas, pero no describe cómo se recopilarán los datos de las entrevistas en profundidad. El proyecto 1990415 explica detenidamente cómo se recogerá la información a través de fuentes documentales y fuentes orales. En relación al análisis de datos, el proyecto 19904159 no expone a qué tipo de análisis serán sometidos los datos o la información recopilada. En cambio, el proyecto 1990431 explicita su forma de análisis, al igual que el proyecto 1990613, que señala que se realizarán dos tipos de análisis de manera bastante detallada.

Sociología 1999. Con respecto al diseño metodológico por disciplina y año, fueron aprobados siete proyectos el año 1999 en el área de sociología. Solo el proyecto 1990392 definió universo, muestras y unidades de análisis. En relación a los otros proyectos, en la investigación 1990818 se indica que se utilizarán fuentes cualitativas y cuantitativas y respecto a este criterio se establecen dos tipos de diseños metodológicos, uno cualitativo y otro cuantitativo. Para el diseño cualitativo no se identifica claramente el primer paso en la propuesta de Vieytes: definir universo, muestras y unidades. Sin embargo, algo de ello se deja entrever cuando se especifica la preparación de instrumentos y recolección de datos, acápite en el que se precisa la cantidad de casos disponibles para el estudio a través de "entrevistas individuales provenientes de estudios ocupacionales o comunitarios en Buenos Aires y Montevideo" y una selección de 90 entrevistas. Con respecto al diseño cuantitativo, en las encuestas se infiere que la unidad de análisis es la familia, con un muestreo aleatorio simple. En el proyecto 1990562 se definen claramente los tres componentes del primer paso de un diseño metodológico según Vieytes: universo, muestras y unidades de análisis. Respecto al proyecto 1990163 no se explicita el universo, las muestras, ni las unidades de análisis. Sin embargo, se puede deducir según lo planteado que la unidad de análisis corresponde "a los varones y mujeres de sectores populares". En relación al proyecto 1990383 se desarrolla extensamente el 
nivel epistemológico y teórico, los niveles de estudio y de análisis que se ocuparán en la investigación. No obstante, no se define de forma clara el universo, muestras y unidades de análisis de la investigación. Se menciona el estudio de ocho comunas sin mencionar sus nombres, de lo que se infiere que puede ser la muestra. Con respecto a las unidades de análisis solo puede ser deducido en la elaboración de los instrumentos, por lo menos para las entrevistas en profundidad de forma precisa, no así en los otros instrumentos siendo más bien ambigua la información. Con respecto al proyecto 1990162 no se señala claramente cada uno de estos tres componentes: universo, muestras y unidades. Sólo se indica que la investigación considera estudiar diez comunas, las que se mencionan, justificando las razones de la elección. Por último, el proyecto 1990140 señala claramente los criterios para la selección de la muestra tanto para las entrevistas como para la conformación de los grupos de discusión.

Respecto al instrumento, el proyecto 1990818 señala que utilizará encuestas, especificándose las categorías que serán medidas. El proyecto 1990562 expone detalladamente la elaboración y el uso de los instrumentos, explicando para cada instrumento cómo se recogerán los datos, además de los objetivos que se persiguen y se explica cómo se llegará a las personas para obtener la información. Con respecto al proyecto 1990163 se recurrirá a datos secundarios, enfatizándose detalladamente el análisis de dicha información. Respecto a cómo se recolectarán los datos se indica que "a través de las declaraciones de los sumarios criminales". En relación al proyecto 1990383 respecto al instrumento se explica claramente y se define el objetivo de cada uno: "encuesta estandarizada y luego en una segunda etapa la observación participante, la entrevista en profundidad y los focus groups", sin embargo, no se da cuenta de la operacionalización de conceptos. El proyecto 1990392 explicita el instrumento y da cuenta de las variables a ser utilizadas "basado en micro y macro data que provendrá de dos instrumentos que seguirán los lineamientos básicos de los Surveys". Con respecto al proyecto 1990162, se señala que se estudiarán documentos secundarios diversos y que tal documentación se relacionará con las categorías de problemas ambientales con el objetivo de detectar áreas y graficar problemáticas homogéneas, lo que da señales de cierta conceptualización y operacionalización de dimensiones o variables. Lo anterior, tiene estrecha relación con la recolección de los datos, pues sobre la base de esta información se realizará "una primera aproximación al problema por medio de la elaboración de planos que identifiquen, por un lado, la pobreza y, por otro, los problemas ambientales del país". El método de superposición de planos permitiría detectar áreas homogéneas en que se articulan la pobreza y el deterioro ambiental. Finalmente, el proyecto 1990140 respecto a los instrumentos indica que se utilizarán: "entrevistas semiestructuradas, grupos de discusión y un cuestionario". Se explicitan los conceptos a ser medidos a través de cada instrumento, pero no operacionalizados. También se indican otras fuentes de información secundaria. Respecto a recolectar los datos se indica solamente para el cuestionario que: "los datos recogidos serán procesados computacionalmente y se traducirán en cuadros". Para el resto de las técnicas, no se indica cómo se recogerá la información.

Con respecto al análisis de los datos, el proyecto 1990818 describe de manera general el análisis con respecto a las encuestas y a las entrevistas. En el proyecto 1990562 se indica claramente el análisis para los datos de carácter cuantitativo y el programa computacional que se utilizará, no sucede lo mismo respecto a los datos de carácter cualitativo. El proyecto 1990163 desarrolla en detalle el análisis de los datos a través del análisis de discurso, y comprendiendo el estudio desde tres niveles. Con respecto al proyecto 1990383 no se señala nada explícitamente sobre el análisis de datos. El proyecto 1990392 señala el software a utilizarse en el procesamiento de los datos, argumentando la idoneidad de los paquetes estadísticos. El proyecto 1990162 respecto a las técnicas de análisis de los datos señala que se realizará "una matriz de interacciones entre pobreza y medio ambiente", lo que permitirá un análisis específico, además de una tipificación de las relaciones entre pobreza y medio ambiente. Finalmente, en el proyecto 
1990140 no se hace ninguna mención al análisis de los datos. Con respecto a la interpretación de los datos, sólo el proyecto 1990163 indica que "el análisis de los resultados se dará respecto a tres dimensiones, las cuales dan respuesta a los tres objetivos específicos de la investigación".

\section{Conclusiones}

Respecto al trabajo metodológico, se puede inferir que la mayoría de las investigaciones, de ambas disciplinas, explicitan el tipo de diseño utilizado, ya sea cuantitativa y/o cualitativa, siendo la sociología la que explicita mayormente. Aparentemente, también podemos concluir que no existe relación entre tipo de diseño utilizado (cualitativo/cuantitativo) y el dar cuenta de manera explícita sobre el uso de ellos.

En términos generales las opciones metodológicas para ambas disciplinas optan por un diseño mixto o sólo cualitativo. La metodología cuantitativa es la menos utilizada en los periodos investigados. Si se comparan ambas disciplinas, la antropología opta por lo cualitativo, en cambio sociología opta principalmente por la combinación de ambos diseños metodológicos.

En términos de disciplina, en los proyectos de antropología menos de la mitad de las investigaciones dan las razones del uso de una metodología. En sociología también menos de la mitad lo hace (cuatro de diez), lo cual devela que a pesar de ser una muestra muy pequeña, en ambas disciplinas pareciera ser que no se tiene incorporado como algo fundamental el explicitar el uso de un determinado diseño metodológico. Lo anterior, puede deberse a las diferencias y polémicas que existen entre una y otra metodología, lo que puede limitar el explicitar las razones, que no se consideren relevante o simplemente no haya una reflexión explícita al respecto.

Respecto a las razones dadas para el uso de un diseño u otro, en general los argumentos dados apuntan principalmente al objeto de estudio, lo cual es coherente con el campo de la investigación y la elección de un tipo de metodología. Esto es de suma importancia, pues más allá de las disciplinas y del uso de un método cualitativo o cuantitativo o una estrategia mixta, la coherencia de una investigación radica principalmente en que su estrategia metodológica se elabore respondiendo a los objetivos que se plantea la investigación.

Sin embargo, hay un par de pasos de la propuesta que son de suma relevancia y que no todos los proyectos cumplían. Por ejemplo, muchas propuestas metodológicas indican los instrumentos, pero muy pocas operacionalizan variables o señalan indicadores. Asimismo varias investigaciones omiten tres cosas fundamentales: muestra, unidad de análisis y análisis de los datos. La mayoría de las investigaciones se centran principalmente en la descripción de los instrumentos o técnicas a utilizar, lo que es una mirada reduccionista a la hora de elaborar una estrategia metodológica de una investigación y que sin duda constituye un argumento para el decaimiento que presentan los estudios e investigaciones en las ciencias sociales.

Debido a lo anterior, podemos concluir que por lo menos en los proyectos analizados, no existe un consenso con respecto a lo mínimo que debe contener un diseño metodológico, lo que se refleja en la variedad de elementos contenidos u omitidos. Por otra parte, se constata que Fondecyt no explicita de manera exhaustiva los elementos básicos que debiera contener el diseño metodológico, lo cual supone que se debe a que los postulantes a estos fondos son investigadores con trayectoria y conocimiento en los aspectos metodológicos. Sin embargo, debido a lo expuesto anteriormente es relevante consensuar criterios mínimos y fundamentales en aspectos tan importantes como el diseño metodológico de cualquier 
tipo y área de investigación, ya que los datos reflejan que no existe tal consenso, ni criterio en común, sobre todo en uno de los organismos más importantes en Chile.

Por otra parte, llama la atención que en un nivel de proyectos Fondecyt (donde uno esperaría mucha minuciosidad y transparencia) se presenten algunos proyectos con diseños metodológicos bastante simples y omitiendo muchos elementos fundamentales y que aun así sean aprobados. Lo que lleva a confirmar lo señalado por Ruth Sautu (2005) en su preocupación frente al desconocimiento o quizás la poca rigurosidad en no señalar elementos fundamentales de cualquier diseño metodológico de los investigadores actuales.

Lo descrito puede explicarse por una formación profesional menos rigurosa, debido a que cada día existe menos reconocimiento a quienes guían el proceso pedagógico de formación de los estudiantes, también puede obedecer a la lógica de mercado que afecta todos los ámbitos de la sociedad y que ha trasformado los procesos de investigación casi en una producción en serie "fordista" que se expresa en priorizar el mayor volumen de publicación, lo que implica cada vez menos tiempo para el proceso de elaboración de las propuestas de investigación, para la reflexión y menos reconocimiento a los procesos de formación. Hoy es más importante la cantidad que la profundidad y el aporte que puedan tener las diversas publicaciones académicas. Esta presión por una producción "desenfrenada" y que se asume por parte de la mayoría de los investigadores, sujetos no ajenos a la dinámicas hegemónicas de las sociedades, obedece, en el mejor de los casos, a un aumento en el sueldo de los investigadores que ya tienen una plaza y para los "nuevos" investigadores se impone como exigencia para poder concursar y competir en mejores condiciones para optar a un trabajo en alguna institución académica, publicaciones que por cierto no aseguran un empleo como investigador. Asimismo, esta situación también impulsa perversamente el llamado "refriteo", que es hacer múltiples publicaciones de la misma investigación, modificando el título u otros pequeños aspectos para responder a las exigencias académicas y poder lograr un espacio en el "mercado" académico.

Para finalizar, solo mencionar que esta dinámica afecta el proceso creativo, de reflexión, de originalidad y de rigurosidad que exige cualquier investigación, ya que requiere de tiempo y maduración si lo que se busca es un aporte al conocimiento y alguna mejora en las condiciones de los sujetos.

\section{Agradecimientos}

Se agradece el diálogo y el aporte que realizó Carla Viera para este trabajo y que están incorporados en la versión final.

\section{Bibliografía}

Briones, G. 1989. Métodos y técnicas avanzadas de investigación aplicadas a la educación y a las ciencias sociales. Santiago: PIIE.

Cook, T. y Reichardt, C. 2005. Métodos cualitativos y cuantitativos en investigación evaluativa. Madrid: Morata.

Hernández, R; Fernández, C. y Baptista, P. 2004. Metodología de la investigación. México: McGraw-Hill.

Pérez, G. 2001. Investigación cualitativa. Retos e interrogantes. Madrid: La Muralla. 
Perelló, S. 2009. Metodología de la investigación social. Madrid: Dykinson.

Sautu, R; Boniolo, P; Dalle, P y Elbert, R. 2005. Manual de metodología. Buenos Aires: CLACSO.

Vieytes, R. 2004. Metodología de la investigación en organizaciones, mercado y sociedad: epistemología y técnicas. Buenos Aires: Editorial de las Ciencias.

Recibido el 12 Dic 2014

Aceptado el 6 Abr 2015 